\title{
s? \\ Boundary criticality at the Anderson transition between a metal and a quantum spin Hall insulator in two dimensions
}

\author{
Hideaki Obuse, ${ }^{1, *}$ Akira Furusaki, ${ }^{1}$ Shinsei Ryu, ${ }^{2}$ and Christopher Mudry ${ }^{3}$ \\ ${ }^{1}$ Condensed Matter Theory Laboratory, RIKEN, Wako, Saitama 351-0198, Japan \\ ${ }^{2}$ Kavli Institute for Theoretical Physics, University of California, Santa Barbara, California 93106, USA \\ ${ }^{3}$ Condensed Matter Theory Group, Paul Scherrer Institute, CH-5232 Villigen PSI, Switzerland \\ (Received 26 May 2008; revised manuscript received 17 July 2008; published 2 September 2008)
}

\begin{abstract}
Static disorder in a noninteracting gas of electrons confined to two dimensions can drive a continuous quantum (Anderson) transition between a metallic and an insulating state when time-reversal symmetry is preserved but spin-rotation symmetry is broken. The critical exponent $\nu$ that characterizes the diverging localization length and the bulk multifractal scaling exponents that characterize the amplitudes of the critical wave functions at the metal-insulator transition do not depend on the topological nature of the insulating state, i.e., whether it is topologically trivial (ordinary insulator) or nontrivial (a $Z_{2}$ insulator supporting a quantum spin Hall effect). This is not true of the boundary multifractal scaling exponents, which we show (numerically) to depend on whether the insulating state is topologically trivial or not.
\end{abstract}

DOI: 10.1103/PhysRevB.78.115301

PACS number(s): 73.20.Fz, 71.70.Ej, 73.43.-f, 05.45.Df

\section{INTRODUCTION}

It has long been known that the metallic state of a twodimensional gas of noninteracting electrons is robust to sufficiently weak static disorder when time-reversal symmetry (TRS) is preserved but spin-rotation symmetry (SRS) is broken. ${ }^{1}$ Increasing the disorder strength relative to the Fermi energy induces a continuous quantum (Anderson) transition to an insulating state. The bulk properties of this metal-insulator transition have been well characterized numerically when the insulating state is topologically trivial. ${ }^{2-4}$

It has been realized only in the last few years that there are two distinct classes of time-reversal-invariant (band) insulators: $\mathbb{Z}_{2}$ topologically trivial and nontrivial insulators. The $Z_{2}$ topological insulator in two dimensions supports a helical edge state which is a Kramers pair of counterpropagating gapless excitations. ${ }^{5,6}$ This helical edge state is responsible for the quantum spin Hall (QSH) effect: An electric field induces a spin accumulation on the edges transverse to the direction of the electric field. ${ }^{5,6}$ The QSH effect has been observed in $\mathrm{HgTe} /(\mathrm{Hg}, \mathrm{Cd}) \mathrm{Te}$ quantum wells. ${ }^{7,8}$ Just as edge states in the integer quantum Hall effect are stable against disorder, the helical edge state in a $\mathbb{Z}_{2}$ topological insulator survives impurity scattering as long as the bulk energy gap is open and the TRS is preserved. ${ }^{9,10}$ This implies that, even in the presence of disorder, time-reversal-invariant insulators can be classified into two distinct classes, $\mathbb{Z}_{2}$ topological and nontopological (ordinary) insulators, according to the presence or absence of a helical edge state.

It is then natural to ask ${ }^{11}$ whether the transition between the metallic and the QSH insulating states belongs to a universality class different from that of the (ordinary) twodimensional symplectic universality class discovered in Ref. 1. It was shown in Ref. 12 that the answer is negative for the scaling exponent $\nu$ of the diverging localization length upon approaching the Anderson transition from the insulating sides. In this paper, we show numerically that there are boundary multifractal scaling exponents ${ }^{13}$ that are sensitive to the presence or absence of a helical edge state on the insulating side of the transition.
We review in Sec. II the definition of the two-dimensional network model introduced in Ref. 12 that encodes the transition between the metallic and QSH insulating states, as well as the transition between the metallic and the ordinary insulating states in the two-dimensional symplectic universality class. The latter transition is conventionally studied using the two-dimensional tight-binding model introduced in Ref. 2, which is also briefly reviewed. The phase diagram for the network model is reviewed, and the relevance of boundary conditions to the presence or absence of helical edge states is discussed in Sec. III. The dependence of the localization length on transverse boundary conditions in quasione-dimensional geometries is discussed in Sec. IV. Boundary multifractal scaling exponents in the network model are calculated numerically in Sec. V. Corner multifractal scaling exponents in the network model are investigated analytically in Sec. VI. We conclude with Sec. VII.

\section{NETWORK MODEL}

Our starting point is a network model introduced in Ref. 12 to capture the Anderson transition between the twodimensional metallic and topological insulating states. The network model is constructed by decorating an underlying square lattice of sites and single bonds connecting nearestneighbor sites with the elementary building blocks from Fig. 1. By taking advantage of the bipartite nature of the square lattice, one colors all sites from one sublattice in red and all sites from the complementary sublattice in blue. One then replaces any red (blue) site with a node $\mathbf{S}\left(\mathbf{S}^{\prime}\right)$ represented graphically with an open red (blue) circle. Second, any single bond connecting a pair of nearest-neighbor sites of the square lattice is replaced with a pair of directed links of opposite orientation. On the links, the spin-1/2 is a good quantum number. A link represented by a full line carries the spin-1/2 quantum number $\sigma=\uparrow$. A link represented by a dashed line carries the spin-1/2 quantum number $\sigma=\downarrow$. Third, the four pairs of directed links that meet at a node are 
(a)

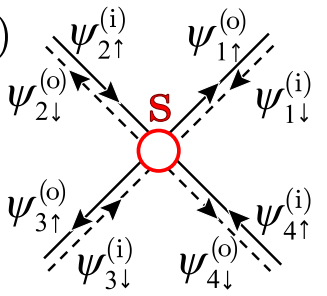

FIG. 1. (Color online) Elementary building blocks of the network model. A square Bravais lattice with nearest-neighbor sites connected by bonds underlies the construction of the network model. Any red site of one of the two sublattices of the square lattice is replaced by a red circle $\mathbf{S}$ (a node of type $\mathbf{S}$ ) with the four bonds meeting at the site replaced by four pairs of directed links numbered according to the rule shown in (a). Any blue site of the complementary sublattice of the square lattice is replaced by a blue circle $\mathbf{S}^{\prime}$ (a node of type $\mathbf{S}^{\prime}$ ) with the four bonds meeting at the site replaced by four pairs of directed links numbered according to the rule shown in (b). Observe that a clockwise rotation by $\pi / 2$ turns (a) into (b). The directed links represent incoming or outgoing plane waves with a well-defined projection of the spin-1/2 quantum number along the quantization axis. Each pair of links replacing a bond represents a Kramers doublet of plane waves. Each node $\mathbf{S}$ or $\mathbf{S}^{\prime}$ depicts a scattering process represented by a $4 \times 4$ unitary matrix defined in Eq. (2.1) that preserves TRS but breaks SRS.

labeled according to the rules in Figs. 1(a) and 1(b) if the node is of types $\mathbf{S}$ and $\mathbf{S}^{\prime}$, respectively. With the conventions in Figs. 1(a) and 1(b), either node defines a $4 \times 4$ scattering matrix $S$ that preserves TRS but breaks SRS and can be represented by

$$
\begin{gathered}
\left(\begin{array}{c}
\psi_{1 \uparrow}^{(o)} \\
\psi_{2 \downarrow}^{(o)} \\
\psi_{3 \uparrow}^{(o)} \\
\psi_{4 \downarrow}^{(o)}
\end{array}\right)=S\left(\begin{array}{l}
\psi_{2 \uparrow}^{(i)} \\
\psi_{1 \downarrow}^{(i)} \\
\psi_{4 \uparrow}^{(i)} \\
\psi_{3 \downarrow}^{(i)}
\end{array}\right), \quad S=\left(\begin{array}{cc}
r \sigma_{0} & t Q \\
-t Q^{\dagger} & r \sigma_{0}
\end{array}\right), \\
r=\tanh X, \quad t=\frac{1}{\cosh X}
\end{gathered}
$$

$$
\begin{aligned}
Q= & i \sigma_{0} \cos \theta \sin \varphi_{1}+\sigma_{1} \sin \theta \cos \varphi_{2}-\sigma_{2} \sin \theta \sin \varphi_{2} \\
& +\sigma_{3} \cos \theta \cos \varphi_{1} .
\end{aligned}
$$

Here, the four matrices $\sigma_{0,1,2,3}$ act on the spin-1/2 components with $\sigma_{0}$ as the unit $2 \times 2$ matrix and $\left(\sigma_{1}, \sigma_{2}, \sigma_{3}\right)$ as the three Pauli matrices. Moreover, $0 \leq X<\infty, 0 \leq \theta<\pi / 2,0$ $\leq \varphi_{1}<2 \pi$, and $0 \leq \varphi_{2}<2 \pi$. TRS is represented by the condition

$$
S=\left(\begin{array}{cc}
\sigma_{2} & 0 \\
0 & \sigma_{2}
\end{array}\right) S^{T}\left(\begin{array}{cc}
\sigma_{2} & 0 \\
0 & \sigma_{2}
\end{array}\right) .
$$

The matrix $S$ is the most general $4 \times 4$ unitary matrix that describes a quantum tunneling process between two Kramers doublets that preserves TRS but can break SRS. When $r=1$ and $t=0, S$ is reduced to the unit matrix, and there is no tunneling between one Kramers pair $\left(\psi_{1}+\psi_{2}\right)$ and the other pair $\left(\psi_{3}+\psi_{4}\right)$. The tunneling with (without) a spin flip occurs with the probability $t^{2} \sin ^{2} \theta\left(t^{2} \cos ^{2} \theta\right)$. Although $S$ is pa-

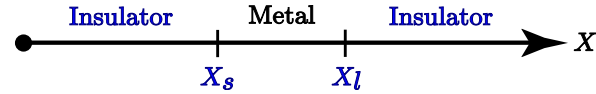

FIG. 2. (Color online) Phase diagram for the network model after Ref. 12 .

rametrized by four real parameters, only $X$ and $\theta$ matter as $\varphi_{1}$ and $\varphi_{2}$ can be absorbed in the overall phase windings that the Kramers doublets acquire when traversing along links connecting nodes.

Disorder is introduced in the network model by assuming that the phases of Kramers doublets on the links and the phases $\theta$ on the nodes are independently and identically distributed. The distribution of the link phase of a Kramers doublet is uniform over the interval $[0,2 \pi]$. The distribution of $\theta$ is $\sin 2 \theta$ over the interval $[0, \pi / 2]$. We are left with one parameter $X$ in the network model that controls the scattering amplitude at every node. The parameter $\theta$ in the network model plays the same role as spin-orbit interactions (of Rashba type) in a random tight-binding Hamiltonian belonging to the two-dimensional symplectic symmetry class, whereas the parameter $X$ plays the role of the Fermi energy.

In order to distinguish the topologically trivial insulating phase from the QSH insulating phase, we shall compare the results that we obtained from the network model against the ones that we obtained from a two-dimensional tight-binding model introduced in Ref. 2, the so-called SU(2) model. The $\mathrm{SU}(2)$ model is a microscopic random tight-binding Hamiltonian with on-site randomness (box distributed with the width $W$ ) and with random hopping amplitudes such that the spin-dependent hopping amplitudes between any pair of nearest-neighbor sites of a square lattice are distributed so as to generate the $\mathrm{SU}(2)$-invariant Haar measure. It captures the transition between a metallic and a topologically trivial insulating state in the ordinary two-dimensional symplectic universality class. In the SU(2) model, the Fermi energy plays the role of the network parameter $X$ for a fixed and not too strong $W$.

\section{PHASE DIAGRAM AND BOUNDARY CONDITIONS}

On symmetry grounds, we expect that it is possible to drive the network model through two successive Anderson transitions by tuning $X$. Indeed, this was shown to be the case in Ref. 12. For any $X$ bounded by the two critical values $X_{s}<X_{l}\left[X_{s}=0.047 \pm 0.001, X_{l}=0.971 \pm 0.001\right.$; the subscript $s$ (l) stands for small (large)] the network model is in a metallic state, while for $X<X_{s}$ or for $X_{l}<X$ it is in an insulating state. It was shown in Ref. 12 that the localization length, a bulk property of any one of the two insulating phases, diverges with the exponent $\nu \approx 2.7$ of the ordinary twodimensional symplectic universality class ${ }^{2}$ upon approaching the mobility edge (either $X_{s}$ or $X_{l}$ ). The resulting phase diagram is shown in Fig. 2. The conclusions from Ref. 12 were reached by studying numerically the network model in a strip geometry with periodic boundary conditions (PBCs) imposed in the transverse direction.

Here, we shall depart from Ref. 12 by imposing reflecting boundary conditions (RBCs) in the transverse direction in 
(a)

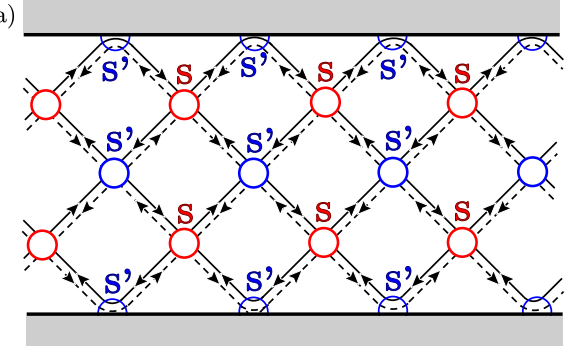

(b)

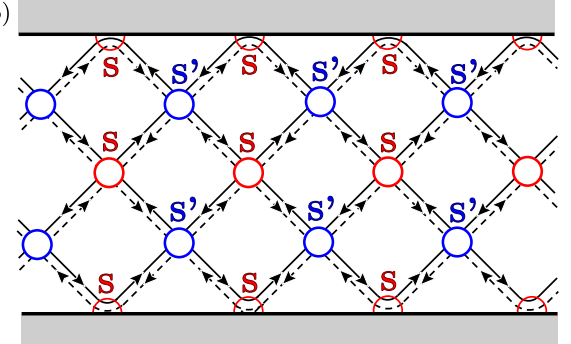

(c)

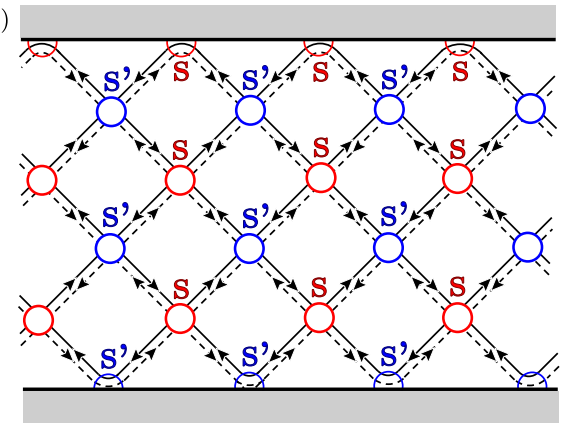

FIG. 3. (Color online) Quasi-one-dimensional network model with reflecting boundaries. (a) The upper and lower boundaries pass through the nodes $\mathbf{S}^{\prime}$. (b) The upper and lower boundaries pass through the nodes $\mathbf{S}$. (c) The upper and lower boundaries pass through the nodes $\mathbf{S}$ and $\mathbf{S}^{\prime}$, respectively. Transverse periodic boundary conditions are possible only in geometries (a) and (b), for which the transverse width is here $M=2$.

the strip geometry such as in Fig. 3 and 4(a), for example. Reflecting boundaries for the network model that respect TRS are defined by choosing a set of nodes and amputating two pairs of links (one labeled by an odd integer and the other by an even integer in the convention of Fig. 1) attached to any of these nodes in a consistent fashion; i.e., no node can be the end point of an odd number of pairs of links. A

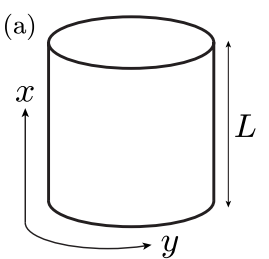

(b)

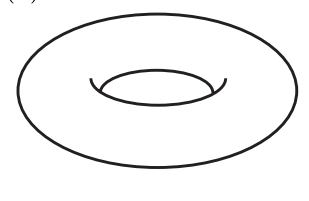

FIG. 4. (a) The network model on a square with $L^{2}$ nodes of type $\mathbf{S}$ and $L^{2}$ nodes of type $\mathbf{S}^{\prime}$ (a node on a boundary is counted as $1 / 2$ ) is wrapped around a cylinder by imposing RBCs along the $x$ direction and PBCs along the $y$ direction. (b) It is wrapped around a torus by imposing PBCs in the $x$ and $y$ directions. (a)

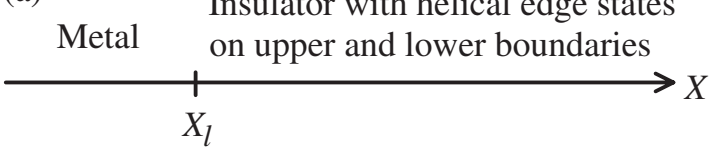

(b)

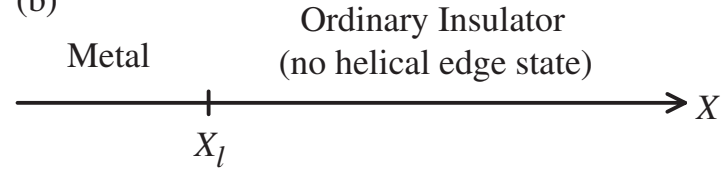

(c)

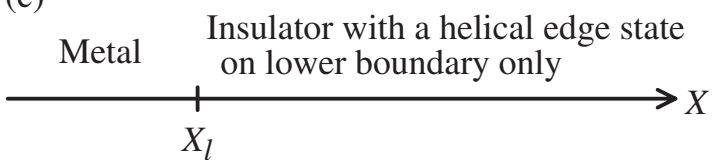

FIG. 5. Phase diagram of the network model of the quasi-onedimensional geometries in Figs. 3(a)-3(c) at $X \gtrsim X_{l}$. The choice of the boundary decides whether the insulating phase at $X>X_{l}$ has helical edge states.

boundary node is depicted by a colored semicircle, and its scattering matrix $S$ is always represented by a unit $2 \times 2$ matrix, regardless of its index $\mathbf{S}$ or $\mathbf{S}^{\prime}$. Three examples of reflecting boundaries defining a strip geometry are shown in Fig. 3. From the fact that the scattering process in Fig. 1(b) is obtained by a clockwise rotation by $\pi / 2$ of the scattering process in Fig. 1(a) follows the important property that a horizontal boundary passing through nodes of type $\mathbf{S}$ is equivalent to a vertical boundary passing through the nodes of type $\mathbf{S}^{\prime}$ and vice versa.

In the following discussions, we shall focus on the critical point $X=X_{l}$ and its neighboring insulating phase. Imposing RBCs, we shall see that the insulating phase at $X>X_{l}$ in the phase diagram from Fig. 2 can acquire a topological attribute in that it supports a single Kramers doublet that is localized in the direction transverse to the boundary but is delocalized along the boundary, i.e., a single Kramers degenerate pair of edge states.

The refined phase diagram in Fig. 5 is depicted for $X \gtrsim X_{l}$ according to the presence or absence of edge states in the insulating phase at $X>X_{l}$. Identifying helical edge states can be done in a pictorial way in the perfect reflection limit $X \rightarrow \infty$ provided we assume continuity of the insulating phase. In the limit $X \rightarrow \infty$, the scattering at the nodes $\mathbf{S}$ and $\mathbf{S}^{\prime}$ reduces to the glancing events depicted in Fig. 6. As a corollary, Fig. 3 simplifies to Fig. 7. We thus see two Kramers doublets propagating along the upper and lower boundaries in Fig. 7(a), none in Fig. 7(b), and one in Fig. 7(c). The refined phase diagrams from Fig. 5 then follow assuming continuity. ${ }^{14}$ In Secs. IV, V, and VI we go beyond the picto- (a)

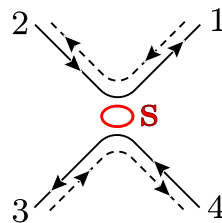

(b) 3

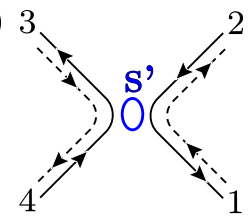

FIG. 6. (Color online) Scattering in the reflective limit $X \rightarrow \infty$ for nodes (a) $\mathbf{S}$ and (b) $\mathbf{S}^{\prime}$. 


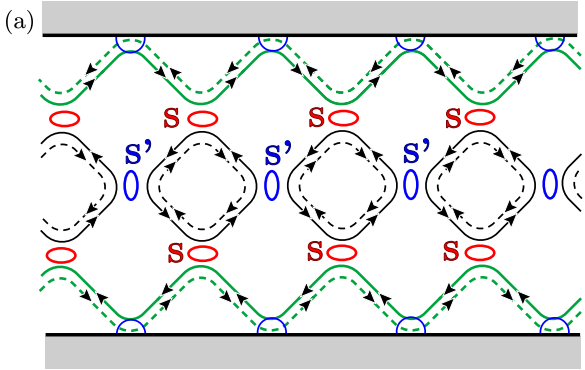

(b)
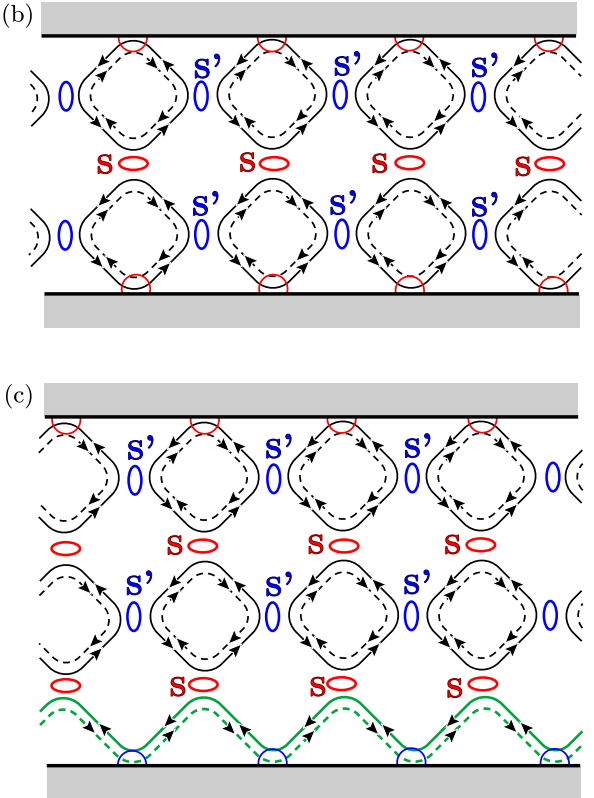

FIG. 7. (Color online) Large $X$ limit of the quasi-onedimensional network model with the reflecting boundaries from Fig. 3.

rial argument in Fig. 7 and study the critical properties at the Anderson metal-insulator transition in a quantitative fashion with an emphasis on the boundary and corner multifractal scaling exponents at criticality.

It is important to note here that we may consider the insulating state in Fig. 5(a) as a $\mathbb{Z}_{2}$ topological insulating state because of the presence of a helical edge state along the boundaries. Similarly, we may identify the insulating state in Fig. 5(b) with a $Z_{2}$ topologically trivial, ordinary insulator. These observations indicate that in the network model formulation, the $\mathbb{Z}_{2}$ topological nature of an insulating state can be determined by appropriate choice of reflecting boundary nodes, even without changing the control parameter $X$. This is very similar to the situation in the Chalker-Coddington network model ${ }^{15}$ for an integer quantum Hall plateau transition, in which an insulating state may or may not have an edge state for a given value of quantum tunneling parameter at nodes, depending on the location of reflecting boundary nodes. An insulating state with an edge state can be regarded as an integer quantum Hall state. We also note that we may attribute this dependence of the topological nature of an insulator on the reflecting boundary conditions in network models to our freedom to give any topological character to the vacuum outside the insulator.

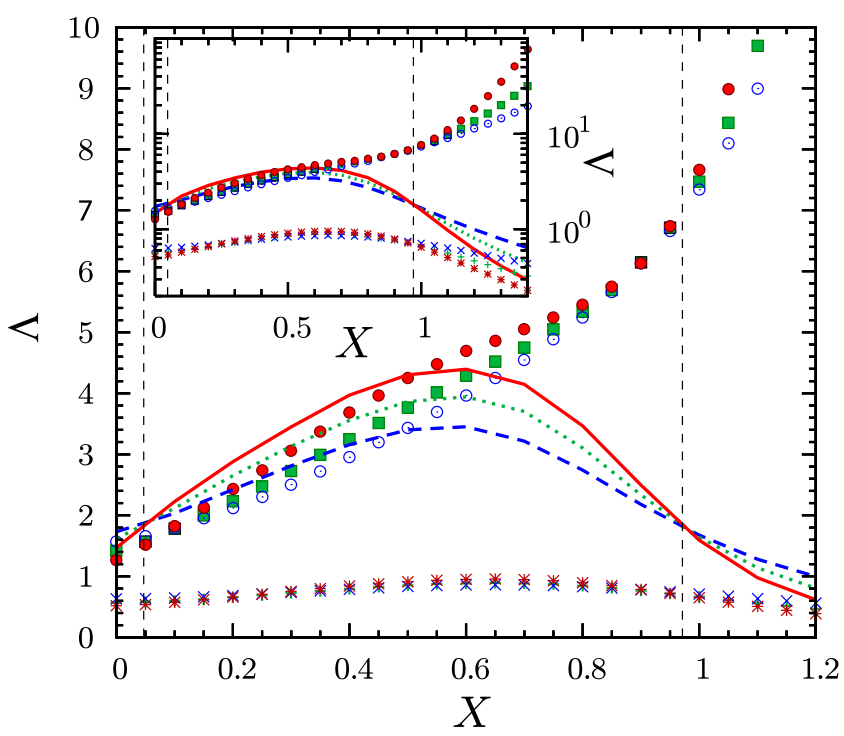

FIG. 8. (Color online) The dependence on $X$ of $\Lambda^{(1)}(X, M)$ in the geometry of Fig. 3(a) with transverse RBCs is shown for $M=4(\odot)$, $8(\square)$, and $16(-)$. The dependence on $X$ of $\Lambda^{(2)}(X, M)$ with transverse RBCs is shown for $M=4(\times), 8(+)$, and $16(*)$. The dependence on $X$ of $\Lambda^{(1)}(X, M)$ with transverse PBCs is shown for $M=4$ (blue dashed curve), 8 (green dotted curve), and 16 (red solid curve). The vertical dashed lines identify the critical points $X_{s}$ and $X_{l}$ deduced in Ref. 12 when transverse PBCs are imposed. The inset displays the same data points with a logarithmic vertical scale.

\section{NORMALIZED LOCALIZATION LENGTH}

Supporting evidence for the phase diagram in Fig. 5(a) can be extracted from the dependence on $M$ of

$$
\Lambda^{(i)}(X, M) \equiv \xi^{(i)}(X, M) / M
$$

in the geometry of Fig. 3(a). In Eq. (4.1), the $i$ th normalized localization length $\Lambda^{(i)}(X, M)$ is the ratio between the $i$ th localization length $\xi^{(i)}(X, M)$, which is given by the inverse of the value of the $i$ th smallest pair ${ }^{16}$ of the Lyapunov exponent of the transfer matrix, and the width $M$ in either one of the geometries in Figs. 3(a) and 3(b). ${ }^{17}$ The transfer matrix for the strip geometries can be constructed in a similar manner as in the cylinder geometry (transverse PBCs). ${ }^{12}$

Figure 8 shows the dependence on $X$ of $\Lambda^{(i)}(X, M)$ with $i=1$ and $i=2$ for the values of $M=4,8$, and 16 in the geometry of Fig. 3(a). Also shown in Fig. 8 is the dependence of $\Lambda^{(1)}(X, M)$ for transverse PBCs. The existence of the critical points $X_{s}$ and $X_{l}$ is signaled by the independence of $\Lambda^{(1)}\left(X_{s, l}, M\right)$ from $M$. Imposing transverse PBCs reduces the finite-size corrections and allows a more accurate measurement of $X_{s}$ and $X_{l}$, the dashed vertical lines in Fig. 8. ${ }^{12}$ When $X_{s}<X<X_{l}, \Lambda^{(i)}(X, M)$ increases with $M$ for both transverse RBCs and transverse PBCs, as expected from a twodimensional metallic state. This trend is reversed in the insulating phases for transverse PBCs, as expected from a twodimensional insulating state. However, when $X_{l}<X$ and transverse RBCs are chosen, $\Lambda^{(1)}(X, M)$ remains an increasing function of $M$, whereas $\Lambda^{(2)}(X, M)$ becomes a decreasing function of $M$ (the inset shows this more clearly with its logarithmic vertical scale). This opposite dependences on $M$ 
of $\Lambda^{(1)}(X, M)$ and $\Lambda^{(2)}(X, M)$ is the signal that each of the upper and lower boundaries supports a single Kramers doublet that would be extended along the boundary ${ }^{18,19}$ were it not for the existence of a finite tunneling amplitude that: (i) couples the two Kramers doublets (edge states) residing near the two opposite boundaries and (ii) is exponentially small in $M$ when $M$ is much longer than the mean free path. The dependence on $M$ of $\Lambda^{(1)}(X, M)$ with transverse RBCs when $X<X_{s}$ is the one expected from an ordinary insulating state. All together, these observations point to the refined phase diagram shown in Fig. 5(a); i.e., the insulating phase is unconventional when $X_{l}<X$ due to the presence of a single Kramers doublet of edge states per boundary that becomes delocalized along the boundaries in the limit $M \rightarrow \infty$.

The $M$-independent normalized localization length at the Anderson transition is nothing but the normalized correlation length $\Lambda_{c}$ at a generic continuous phase transition in the theory of critical phenomena. This quantity is known to depend on the choice of the transverse boundary conditions. ${ }^{20}$ First, the value

$$
\Lambda^{(\mathrm{PBC})}\left(W_{c}\right)=1.844 \pm 0.002
$$

of the normalized localization length at the Anderson transition was calculated in Ref. 2 for the SU(2) model with transverse PBCs. It should here be compared with the values

$$
\begin{aligned}
& \Lambda^{(\mathrm{PBC})}\left(X_{s}\right)=1.81 \pm 0.01, \\
& \Lambda^{(\mathrm{PBC})}\left(X_{l}\right)=1.82 \pm 0.01
\end{aligned}
$$

calculated in Ref. 12. Second, we have extended the calculation in Ref. 2 for the SU(2) model in which transverse PBCs were used to the case in which the vanishing of the wave functions along the transverse boundaries is imposed, a situation that we shall refer to as fixed boundary conditions (FBCs), and found [see Fig. 9(c)]:

$$
\Lambda^{(\mathrm{FBC})}\left(W_{c}\right)=1.50 \pm 0.03 \text {. }
$$

This value should be compared with

$$
\Lambda^{(\mathrm{RBC})}\left(X_{s}\right)=1.49 \pm 0.02
$$

for the network model with transverse RBCs [where we can also deduce the right-hand side from Fig. 9(c) for which it is the network model at the critical point $X_{l}$ in the geometry of Fig. 3(b) that is investigated; Fig. 9(a) shows insulating behavior of $\Lambda^{(1)}$ for $X>X_{l}$ ]. Evidently, values (4.4) and (4.5) agree within their error bars (in support of our identification, made at the end of Sec. III, of the insulating phase without a helical edge state with a $Z_{2}$ topologically trivial insulator) but clearly differ from values (4.2) and (4.3). Finally and more importantly, there is a rather large asymmetry

$$
\Lambda^{(\mathrm{RBC})}\left(X_{l}\right) / \Lambda^{(\mathrm{RBC})}\left(X_{s}\right) \approx 4.8
$$

for the network model with transverse RBCs in the geometry of Fig. 3(a).

Finite-size scaling in Fig. 9(c) clearly shows that the scaling function obtained for the $\mathrm{SU}(2)$ model with transverse FBCs is identical to that for the network model at $X=X_{l}$ in the geometry of Fig. 3(b). These scaling functions are therefore a universal property of the critical point between a me-
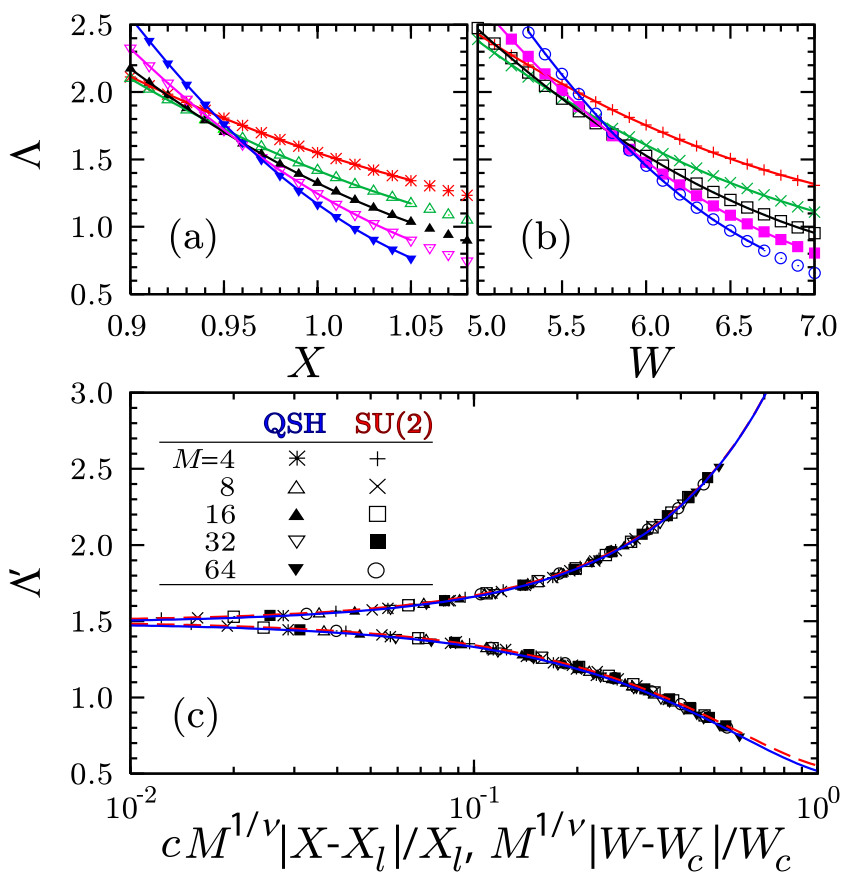

FIG. 9. (Color online) (a) Dependence on $X$ of the normalized localization length $\Lambda^{(1)}(X, M) \equiv \Lambda(X, M)$ for the network model in the quasi-one-dimensional geometry of Fig. 3(b) with transverse RBCs and $M=4,8,16,32,64$. (b) Dependence on the dimensionless disorder strength $W$ of the normalized localization length $\Lambda^{(1)}(W, M) \equiv \Lambda(W, M)$ for the $\mathrm{SU}(2)$ model in the quasi-one-dimensional geometry with transverse $\mathrm{FBCs}$ and $M$ $=4,8,16,32,64$. The solid curves are computed from the finite-size scaling functions that follow. (c) Finite-size scaling analysis for the data shown in (a) and (b): The solid blue curves represent the scaling functions for the network model with RBCs on node $\mathbf{S}$ in the vicinity of the critical point $X_{l}$, at which we find $\Lambda^{(\mathrm{RBC})}\left(X_{l}\right)$ $=1.49 \pm 0.02$ and $\nu=2.88 \pm 0.04$. The red dashed curves represent the scaling functions for the $\mathrm{SU}(2)$ model with FBCs in the vicinity of the critical point $W_{c}$, at which we find $\Lambda^{(\mathrm{RBC})}\left(W_{c}\right)=1.50 \pm 0.03$ and $\nu=2.85 \pm 0.06$. The scaling functions are obtained from finitesize scaling analysis incorporating corrections from a leading irrelevant scaling variable (Refs. 12 and 21). The normalized localization length $\Lambda^{\prime}$ is obtained from $\Lambda$ by subtracting these corrections, as defined in Eqs. (3.7) and (3.8) of Ref. 12. The distance to the critical point $\left|X-X_{l}\right|$ is rescaled by a factor $c \simeq 1.7$ in the scaling function for the network model with RBCs so that it coincides with that for the SU(2) model with FBCs.

tallic phase and an ordinary insulator. From this finite-scaling analysis, we also obtained the critical exponent $\nu$ of the diverging localization length. At the critical point $X_{l}$ of the network model, we found $\nu=2.88 \pm 0.04$. Again, this should be compared with the critical exponent $\nu$ of the SU(2) model with transverse FBCs for which we find $\nu=2.85 \pm 0.06$. Both exponents agree with each other within their error bars; they are also consistent with the exponent $2.7 \lessgtr \nu \leqq 2.8$ obtained with transverse PBCs. ${ }^{2,12}$ This implies that the exponent $\nu$ is a bulk property independent of boundary conditions (whereas the scaling functions are dependent on the transverse boundary conditions).

The network and SU(2) models in a quasi-onedimensional cylinder geometry at criticality are indistin- 
guishable as measured by normalized localization lengths (4.2) and (4.3), respectively. The same is true in a strip geometry provided the insulating side of the transition in the network model is topologically trivial (i.e., has no helical edge states) according to Eqs. (4.4) and (4.5). However, the strong asymmetry [Eq. (4.6)] hints at the possibility that some boundary critical exponents might be sensitive to the choice of transverse boundary conditions that dictates the presence or absence of a delocalized Kramers doublet of edge states.

\section{BULK AND BOUNDARY CRITICALITY}

\section{A. Typical spatial profile of wave functions}

To gain more insights into the criticality of the network model, we study numerically the critical normalized wave functions $\Psi$ when $X=X_{l}$ in the geometries of Figs. 4(a) and 4(b). To this end, the support of normalized wave functions for the network model is defined on the midpoints $(x, y)$ of every bond joining nearest-neighbor nodes; i.e., the normalized wave function $\Psi$ can be viewed as a complex-valued vector whose $8 L^{2}$ components $\psi_{\sigma}(x, y)$, with the spin-1/2 quantum number labeled by $\sigma=\uparrow, \downarrow$, correspond to $4 L^{2}$ freely propagating Kramers pairs of plane waves. The dynamics of such a wave function is governed by a unitary evolution operator $U$ built out of all scattering matrices at the nodes of the network. ${ }^{22}$ For each realization of the disorder, we numerically diagonalize $U$ and retain one normalized wave function whose eigenvalue is closest to 1 . The number of disorder realizations is $5 \times 10^{4}$ for each system size $L(L$ ranging from 20 to 80 ). By tuning the parameter $X=X_{l}$ at each node, this normalized wave function is, with a slight abuse of language, called critical.

First, we define

$$
\left\langle\ln |\Psi|^{2}\right\rangle_{x, L} \equiv(2 L)^{-1} \sum_{y=1}^{2 L} \overline{\ln \left(\sum_{\sigma=\uparrow, \downarrow}\left|\psi_{\sigma}(x, y)\right|^{2}\right)},
$$

where $X$ is tuned to the critical point $X_{l}$ for the network model. The overbar denotes disorder averaging over the nodes through the independently distributed angles $\theta$ as well as over the links through the independently and uniformly distributed random phases of Kramers doublets.

Figure 10 shows the $x$ dependence of $\left\langle\ln |\Psi|^{2}\right\rangle_{x, L}$ for different values of $L$ at the critical point $X_{l}$ in the cylinder geometry of Fig. 4(a). The two figures correspond to different RBCs in the $x$ direction: (a) the boundaries passing through the $\mathbf{S}$ nodes as in Fig. 3(b) and (b) the boundaries passing through the $\mathbf{S}^{\prime}$ nodes as in Fig. 3(a). The boundary bonds are located at $x=1$ and $x=2 L$. At the critical point between the metallic and the insulating states without helical edge state [Fig. 10(a)], the dependence of $\left\langle\ln |\Psi|^{2}\right\rangle_{x, L}$ on $x$ is symmetric about $x=L$, at which it reaches a maximum, and reaches a minimum close to the boundaries. For comparison, we show in Fig. 10(a) $\left\langle\ln |\Psi|^{2}\right\rangle_{x, L}$ of the SU(2) model, in which the on-site disorder strength $W$ is tuned to the critical point $W_{c}$, and the overbar in Eq. (5.1) denotes disorder averaging over the on-site energies and the Haar measure of the hopping matrix elements. We find no qualitative difference in
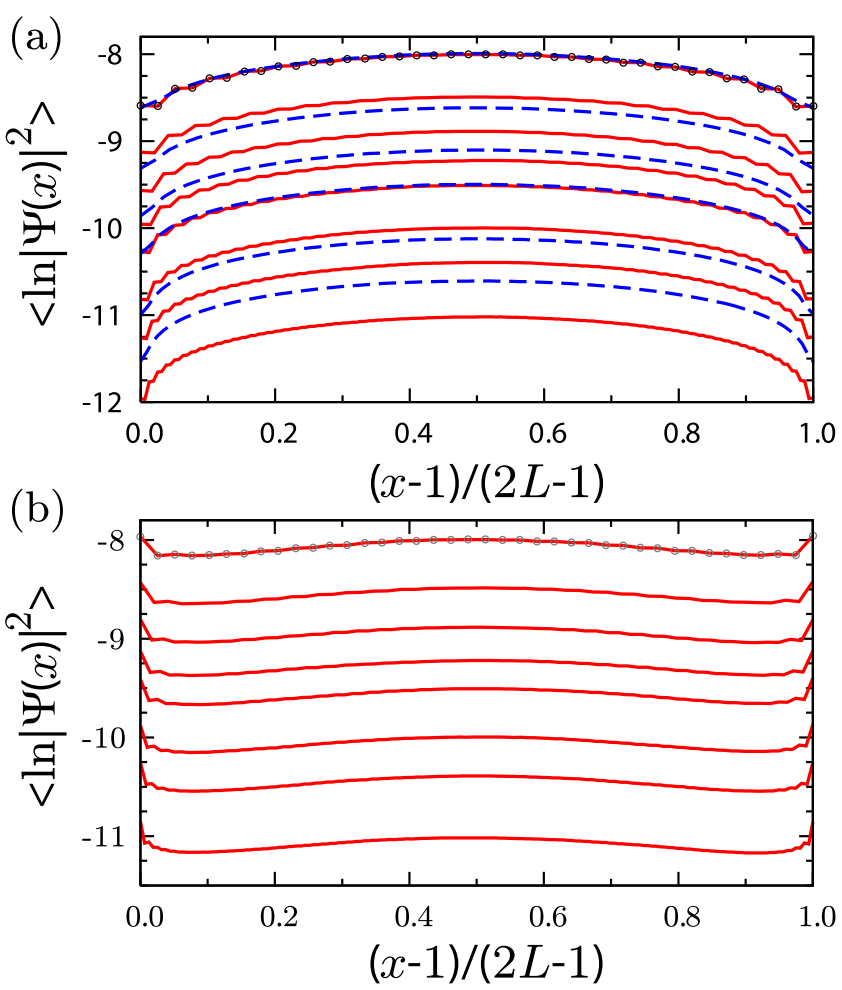

FIG. 10. (Color online) Dependence on the position $x$ along the cylinder axis of Fig. 4(a) of $\left\langle\ln |\Psi|^{2}\right\rangle_{x, L}$ defined by Eq. (5.1) for the values of $L=20,25,30,35,40,50,60,80$ from top to bottom with (a) $\mathbf{S}$ boundaries or with (b) $\mathbf{S}^{\prime}$ boundaries. The blue dashed curves in (a) represent the normalized critical wave functions $\left\langle\ln |\Psi|^{2}\right\rangle_{x, L}$ for $L=36,48,60,72,96,120$ obtained from the SU(2) model defined in Ref. 2 using the cylinder geometry of Fig. 4(a).

the dependence on $x$ and $L$ of $\left\langle\ln |\Psi|^{2}\right\rangle_{x, L}$ between the two models when the critical point separates a metal and an ordinary insulator. Remarkably, Fig. 10(b) shows that the dependence on $x$ of $\left\langle\ln |\Psi|^{2}\right\rangle_{x, L}$ is nonmonotonic when approaching a boundary from the center of the bulk, $x=L$, and that its absolute maximum is reached at the boundaries $x=1,2 L$ instead of the local maximum at $x=L$.

\section{B. Scaling exponents $\Delta_{q}$}

This striking difference between Figs. 10(a) and 10(b) suggests that the statistics of the critical normalized wave functions is sensitive to the topological nature (i.e., the presence or absence of helical edge states) of the insulating state. A powerful tool to study the statistics of critical normalized wave functions is the multifractal scaling analysis. ${ }^{23}$ Multifractality is encoded by a set of scaling exponents (anomalous dimensions) $\Delta_{q}^{(\kappa)}$ defined by the scaling laws

$$
\overline{|\Psi(\boldsymbol{r})|^{2 q}} /\left(\overline{|\Psi(\boldsymbol{r})|^{2}}\right)^{q} \propto L^{-\Delta_{q}^{(\kappa)}}
$$

for $L \gg 1$, where $\Psi$ is the normalized spinor obtained from the network model in the geometry of Figs. 4(a) and 4(b) at criticality $X=X_{l}$, and $q$ is any real number. The index $(\kappa)$ distinguishes bulk, boundary, and corner exponents through the choice of the location of $\boldsymbol{r}=(x, y)$ relative to the boundaries and the nature of the boundaries..$^{3,13,24-26}$ In this paper, 
we distinguish six cases, $(\kappa)=(2),\left(1, \mathbb{Z}_{2}\right),(1,0),\left(0, \mathbb{Z}_{2}\right)$, $(0, \mathrm{O})$, and $\left(0, \mathbb{Z}_{2} \mid \mathrm{O}\right)$. Here the first entry in $(\kappa)$ refers to the dimensionality of the support of wave functions. The second entry in $(\kappa), Z_{2}$ or $\mathrm{O}$ ("ordinary"), refers to the presence or absence, on the insulating side of the critical point, of a Kramers doublet (helical) edge state along a boundary or at a corner where measurement (5.2) is made (see below for more detailed definitions).

In Eq. (5.2) the proportionality constant may have a dependence on $L$ that is much milder than the power law it multiplies. This means that the scaling exponents $\Delta_{q}^{(\kappa)}$ are obtained from calculating numerically

$$
D_{q}^{(\kappa)}(L) \equiv \frac{q \ln \mid \overline{\overline{\left.\Psi(\boldsymbol{r})\right|^{2}}}-\ln \overline{\overline{|\Psi(\boldsymbol{r})|^{2 q}}}}{\ln L}
$$

first. Here, the double overbar means that in addition to the disorder averaging a spatial average over the relevant sites $\boldsymbol{r}$ ( $4 L^{2}$ sites for the torus geometry, $2 L$ sites for the boundary) is also taken to improve the statistics. This is followed by a linear fit of $D_{q}^{(\kappa)}(L)$ as a function of $1 / \ln L$. Only system sizes larger than $L=35$ are kept for the linear fit. At last, $\Delta_{q}^{(\kappa)}$ is obtained as the intercept of the linear fit with the vertical axis at $1 / \ln L=0$. For the case of boundary scaling exponents, we also replaced $|\Psi(\boldsymbol{r})|^{2}$ with $\boldsymbol{r}$ a bond joining a node on the boundary by averaging it over all bonds from the elementary plaquette to which it belongs. This coarse graining is necessary to overcome the small oscillations that are visible in the $x$ dependence plotted in Fig. 10 close to the boundaries. Figure 11 shows the $q$ dependence of the scaling exponents $\Delta_{q}^{(\kappa)}$ for the network and the $\mathrm{SU}(2)$ model in different geometries.

The bulk scaling exponents, for which case $(\kappa)=(2)$, characterize scaling law (5.2) in the torus geometry from Fig. 4(b) (which has no boundary). This is also the case in the cylinder geometry of Fig. 4(a), if the distance from the site $\boldsymbol{r}$ to the boundaries is of order $L$. The bulk exponents shown in Fig. 11(a) are obtained with the torus geometry in order to be free from boundary effects and to maximize the number of sampling points $\boldsymbol{r}$. Figure 11(a) shows that the bulk scaling exponents $\Delta_{q}^{(2)}$ of the network model are identical to those obtained for the SU(2) model $^{3}$ within their error bars.

The boundary scaling exponents are obtained by putting $\boldsymbol{r}$ on the boundaries (with the coarse graining mentioned above) in the cylinder geometry of Fig. 4(a). There is an important caveat however. As we saw in Secs. III and IV, the network model supports two types of (straight) boundaries, while the SU(2) model has only one. Indeed, for the network model at $X=X_{l}$ studied numerically in Fig. 11 we have two cases:

(i) $(\kappa)=(1, \mathrm{O})$, when the boundary on which $\boldsymbol{r}$ is located passes through the nodes of type $\mathbf{S}$. To reduce statistical errors, we take the cylinder geometry of Fig. 4(a) which follows from imposing longitudinal PBCs in Fig. 3(b). No helical edge state exists on the boundaries in the insulating phase at $X>X_{l}$.

(ii) $(\kappa)=\left(1, \mathbb{Z}_{2}\right)$, when the boundary on which $\boldsymbol{r}$ is located passes through the nodes of type $\mathbf{S}^{\prime}$. To reduce statistical errors, we take the cylinder geometry of Fig. 4(a) which
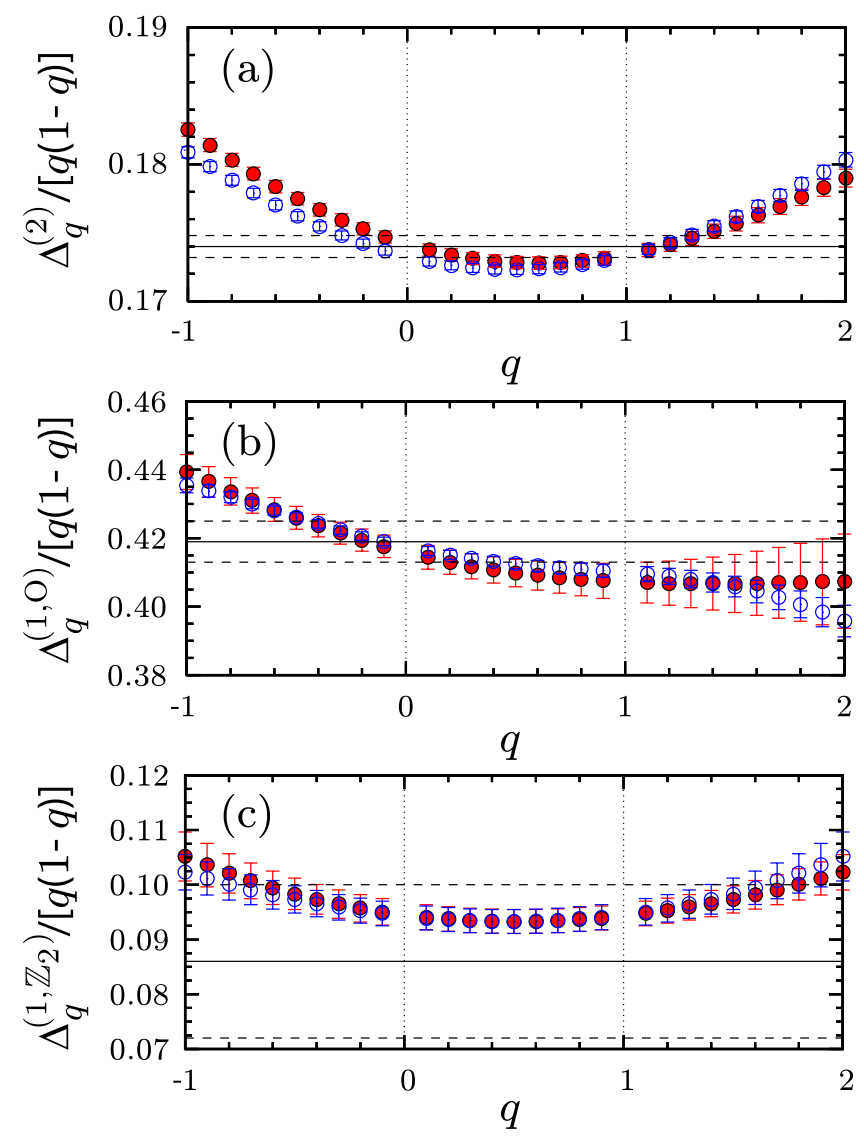

FIG. 11. (Color online) The dependences on $q$ of $\Delta_{q}^{(\kappa)} /[q(1$ $-q)](\mathbf{O})$ when $X=X_{l}$ for (a) the bulk: $(\kappa)=(2)$; (b) the $\mathbf{S}$ boundary: $(\kappa)=(1, \mathrm{O})$; and (c) the $\mathbf{S}^{\prime}$ boundary: $(\kappa)=\left(1, \mathbb{Z}_{2}\right)$. The solid lines represent $\alpha^{(\kappa)}(q)-2$ evaluated at $q=0$ according to Eq. (5.4a) with its error bars indicated by dashed lines. The dependences on $q$ of $\Delta_{q}^{(\kappa)} /[q(1-q)]$ for the $\mathrm{SU}(2)$ model in the bulk and on the boundary are shown with $\bigcirc$ in (a) and (b), respectively. The blue open circles in (c) show the $q$ dependence of $\Delta_{1-q}^{\left(1, Z_{2}\right)} /[q(1-q)]$.

follows from imposing longitudinal PBCs in Fig. 3(a). There exists a helical edge mode on each boundary in the insulating phase at $X>X_{l}$.

Figure 11(b) shows that the boundary scaling exponents $\Delta_{q}^{(1,0)}$ in the network model agree, within their error bars, with the boundary scaling exponents obtained in Ref. 3 from the SU(2) model in the cylinder geometry of Fig. 4(a). Remarkably, Fig. 11(c) shows a second set of boundary scaling exponents $\Delta_{q}^{\left(1, Z_{2}\right)}$ in the network model, which is markedly different from the first set of boundary scaling exponents $\Delta_{q}^{(1,0)}$ in Fig. 11(b). To sum up, the three distinct sets of scaling exponents $\Delta_{q}^{(2)}, \Delta_{q}^{(1, O)}$, and $\Delta_{q}^{\left(1, Z_{2}\right)}$ are identified from Figs. 11(a)-11(c), respectively.

The emerging picture is that the network and the SU(2) models at criticality share common bulk scaling exponents $\Delta_{q}^{(2)}$ and a set of boundary scaling exponents $\Delta_{q}^{(1, \mathrm{O})}$ at an ordinary boundary that does not support a helical edge mode in the neighboring insulating phase. Only the network model has one more set of boundary scaling exponents $\Delta_{q}^{\left(1, Z_{2}\right)}$ at a boundary that has a helical edge mode in the insulating phase. 


\section{C. $f(\alpha)$ spectra}

There is yet another set of scaling exponents $f^{(\kappa)}\left(\alpha^{(\kappa)}\right)$ that we have calculated for the $\mathrm{SU}(2)$ and network models. These exponents are calculated numerically from the scaling Ansätze

$$
\frac{\overline{\overline{|\Psi(\boldsymbol{r})|^{2 q} \ln |\Psi(\boldsymbol{r})|^{2}}}}{\overline{\overline{|\Psi(\boldsymbol{r})|^{2 q}}}} \sim-\alpha_{q}^{(\kappa)} \ln L
$$

and

$$
\ln \overline{\overline{|\Psi(\boldsymbol{r})|^{2 q}}} \sim\left[f^{(\kappa)}\left(\alpha_{q}^{(\kappa)}\right)-\alpha_{q}^{(\kappa)} q-d^{(\kappa)}\right] \ln L,
$$

where $d^{(\kappa)}=2$ and 1 for the bulk and boundary exponents, respectively. It can be shown that the Legendre transform ${ }^{3,13,24-26}$

$$
f^{(\kappa)}\left(\alpha^{(\kappa)}\right) \equiv\left(\alpha^{(\kappa)}-2\right) q-\Delta_{q}^{(\kappa)}+d^{(\kappa)},
$$

where $q$ is a function of $\alpha^{(\kappa)}$ obtained from inverting

$$
\alpha^{(\kappa)}(q)-2 \equiv d \Delta_{q}^{(\kappa)} / d q
$$

relates scaling exponents $(5.2),(5.4 \mathrm{a})$, and (5.4b). The number $f^{(\kappa)}\left(\alpha^{(\kappa)}\right)$ is the fractal (i.e., Hausdorff ${ }^{27}$ ) dimension of the set of points $\boldsymbol{r}$ such that $|\Psi(\boldsymbol{r})|^{2}$ scales as $L^{-\alpha^{(\kappa)}}$.

The dependence on $\alpha^{(\kappa)}$ of $f^{(\kappa)}$ for the critical network model at $X=X_{l}$ in the torus, $(\kappa)=(2)$; in the cylinder geometry with $\mathbf{S}$ boundaries [Fig. $3(\mathrm{~b})],(\kappa)=(1, \mathrm{O})$; and in the cylinder geometry with $\mathbf{S}^{\prime}$ boundaries [Fig. 3(a)], $(\kappa)=\left(1, \mathbb{Z}_{2}\right)$, are shown in Fig. 12(a), which are obtained by combining $\alpha_{q}^{(\kappa)}$ in Fig. 12(b) and $f_{q}^{(\kappa)}$ in Fig. 12(c). We see that there are three distinct multifractal spectra at the critical point $X_{l}: f^{(2)}$ for the bulk, $f^{(1,0)}$ for the $\mathbf{S}$ cylindrical geometry, and $f^{\left(1, Z_{2}\right)}$ for the $\mathbf{S}^{\prime}$ cylindrical geometry. They are compared with the multifractal spectra for the SU(2) model in the torus and cylinder geometry obtained in Ref. 3. Within their error bars, they agree with $f^{(2)}$ and $f^{(1,0)}$, respectively. The exponent $\alpha_{q}^{(\kappa)}$ at $q=0$ is closely related to Eq. (5.1) with $x$ chosen in the bulk or on a boundary. For the network model we find the values

$$
\begin{gathered}
\alpha_{0}^{(2)}=2.174 \pm 0.001, \\
\alpha_{0}^{(1, O)}=2.420 \pm 0.005, \\
\alpha_{0}^{\left(1, Z_{2}\right)}=2.086 \pm 0.015 .
\end{gathered}
$$

The inequality $\alpha_{0}^{\left(1, Z_{2}\right)}<\alpha_{0}^{(2)}<\alpha_{0}^{(1,0)}$ is consistent with the $x$ dependence seen at the boundaries in Fig. 10, as wave functions near $\mathbf{S}^{\prime}$ boundary in the $\mathbf{S}^{\prime}$ cylindrical geometry are expected to be more extended because of the existence of edge modes in the insulating side. We note that the error bars are an order of magnitude larger for $\alpha_{0}^{\left(1, Z_{2}\right)}$ because of the presence of larger finite-size corrections. Within their error bars, values (5.6a) and (5.6b) agree with the ones for the $\mathrm{SU}(2)$ model in the bulk and boundaries, ${ }^{3,4}$ respectively.

It is worth mentioning that the multifractal analysis performed here involves extracting scaling exponents after performing the disorder averaging. Extracting scaling exponents before performing the disorder averaging yields typical scal-
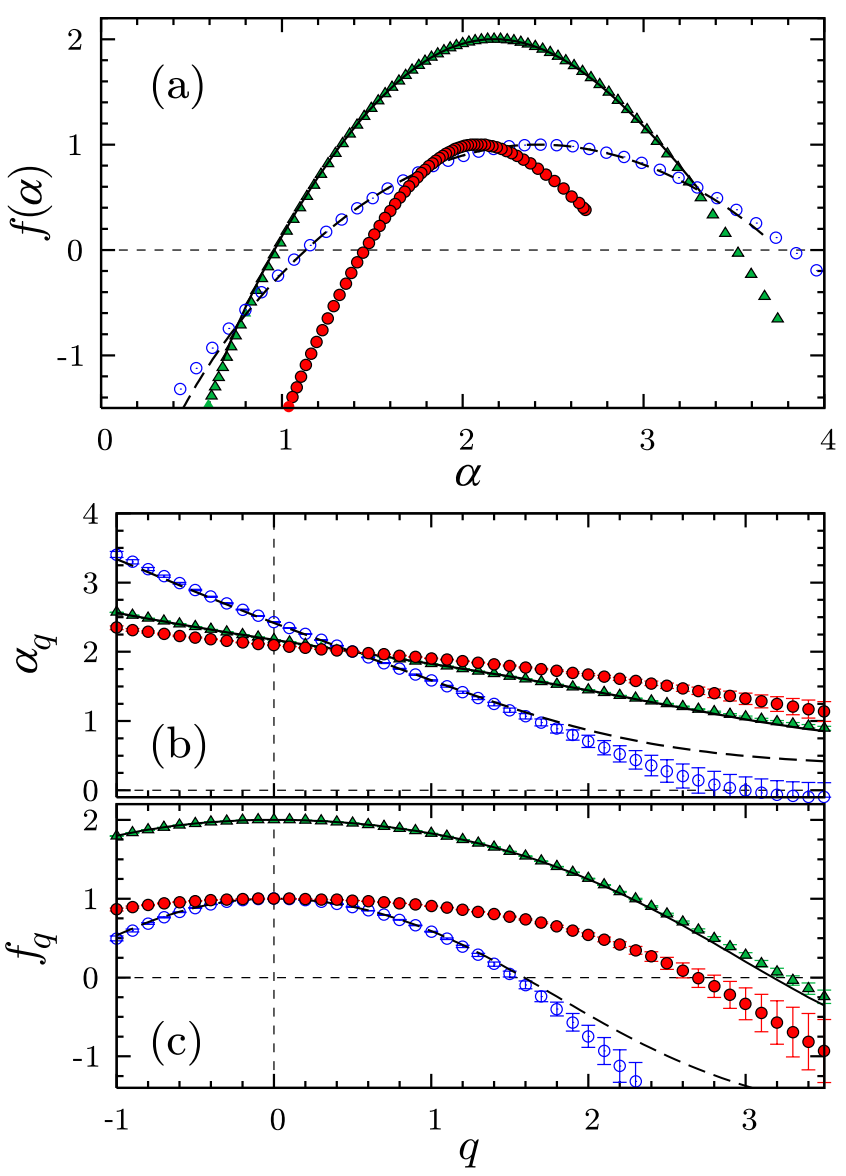

FIG. 12. (Color online) (a) Dependences on $\alpha^{(\kappa)}$ of the multifractal spectra $f^{(\kappa)}$ in the bulk $(\kappa)=(2)(\boldsymbol{\Delta})$, at the $\mathbf{S}$ boundaries $(\kappa)=(1, \mathrm{O})(\odot)$, and at the $\mathbf{S}^{\prime}$ boundaries $(\kappa)=\left(1, \mathbb{Z}_{2}\right)(\mathbf{\odot})$, at $X$ $=X_{l}$. Dependences on $q$ of $\alpha_{q}$ and $f_{q}$ are shown in (b) and (c), respectively, with the same symbols as in (a). The bulk and boundary multifractal spectra for the SU(2) model defined in Ref. 2 are shown as solid and dashed curves, respectively.

ing exponents. Typical scaling exponents need not be identical to average scaling exponents calculated here. ${ }^{28-30}$ The average scaling exponents $\Delta_{q}^{(\kappa)}$ are expected to differ from the typical ones for any values of $q$ such that $f^{(\alpha)}\left(\alpha^{(\kappa)}\right)$ is negative $;^{31}$ see Fig. 12(c). For this range of $q$, rare events dominate the calculation of $\Delta_{q}^{(\kappa)}$ as evidenced by the larger error bars in Fig. 11. This explains the systematic deviations from the mirror symmetry ${ }^{32}$ about $q=1 / 2$ of $\Delta_{q}^{(\kappa)} /[q(q-1)]$ for large $q$ in Fig. 11 .

The rationale for studying these average scaling exponents is that they are expected to be the scaling dimensions of some primary operators representing the moments of wave-function amplitudes in an underlying two-dimensional conformal field theory. ${ }^{33}$ Knowing them constrains the possible field theories that can encode critical properties of an Anderson transition.

\section{CORNER MULTIFRACTALITY}

So far we have always considered geometries of the network model with boundaries of the same type, as in Figs. 
3(a) and 3(b). We are now going to investigate the case of mixed boundaries.

We begin with the network model in the quasi-onedimensional geometry (quantum wire geometry) from Fig. 3(c), for which we show that the Landauer conductance obeys a statistical distribution that differs from that of an ordinary symplectic quantum wire, i.e., a symplectic quantum wire in a geometry compatible with transverse PBCs. A physical realization of this case is given by metallic carbon nanotubes with spatially smooth disorder potential. As shown by Ando and Suzuura, ${ }^{34}$ in the absence of intervalley scattering by disorder, conduction of electronic states near a Fermi point is described by transfer matrices in the symplectic class with an odd number of conduction channels. A mathematical treatment of such conduction process was initiated by Zirnbauer ${ }^{18}$ and further developed by Takane. ${ }^{19}$

We then turn our attention to a semi-infinite geometry with two-point contacts to ideal reservoirs attached at the interface between the boundary of type $\mathbf{S}$ and of type $\mathbf{S}^{\prime}$ as shown in Fig. 13(a). After tuning the network model to criticality, we shall see that this amounts to studying corner multifractal scaling exponents whose values differ from those taken by the corner multifractal scaling at the corner along a homogeneous segment of a boundary; see Fig. 13(b). Common to both examples of mixed boundaries is the existence of a single perfectly conducting channel.

\section{A. Quasi-one-dimensional wire with mixed transverse open boundary conditions}

In the quasi-one-dimensional wire depicted in Fig. 3(c), whereby the length of the disordered region $N$ is larger than the mean free path $\ell \gg 1$, one transmission eigenvalue is always unity for any disorder configuration, while the other $2 M$ eigenvalues are exponentially small with $N$. This follows from applying the results from Refs. 19 and 34 . The same is true of the thick quantum wire limit $N, M \rightarrow \infty$ with the ratio $M \ell / N$ held fixed, as it is now the analysis from Ref. 18 that can be borrowed.

The persistence of the unit eigenvalue of the transmission matrix is unique to the network model with mixed boundary conditions. This remarkable property can be understood physically as follows: Because of the mixed boundaries, one and only one edge supports a single Kramers doublet of edge states. This Kramers doublet of edge states is free to propagate along the edge and thus provides a single perfectly conducting channel. This physics is indeed realized with helical edge modes of a two-dimensional quantum spin Hall insulator and a metallic carbon nanotube in the absence of intervalley scattering. Mathematically, the transfer matrix $\mathcal{M}$ for the geometry of Fig. 3(c) is a member of the group SO $*(4 M+2) .{ }^{12}$ The eigenvalues of $\ln \left(\mathcal{M} \mathcal{M}^{\dagger}\right)$ are symmetrically distributed around 0 and denoted by $\pm 2 x_{i}\left(0 \leq x_{1} \leq x_{2}\right.$ $\left.\leq \cdots \leq x_{2 M+1}\right)$. In turn, the transmission eigenvalues $T_{i}$ are written as $T_{i}=1 / \cosh ^{2} x_{i}(i=1,2, \ldots, 2 M+1)$. Because of the Kramers degeneracy, the eigenvalues of $\ln \left(\mathcal{M M}^{\dagger}\right)$ are twofold degenerate. This leads to the conclusion that there must be a null eigenvalue $x_{1}=0$; thereby $T_{1}=1$.

\section{B. Two-point contacts on a boundary of mixed type}

Next, we consider the case of a semi-infinite network model with a boundary that is mixed, whereby it is necessary

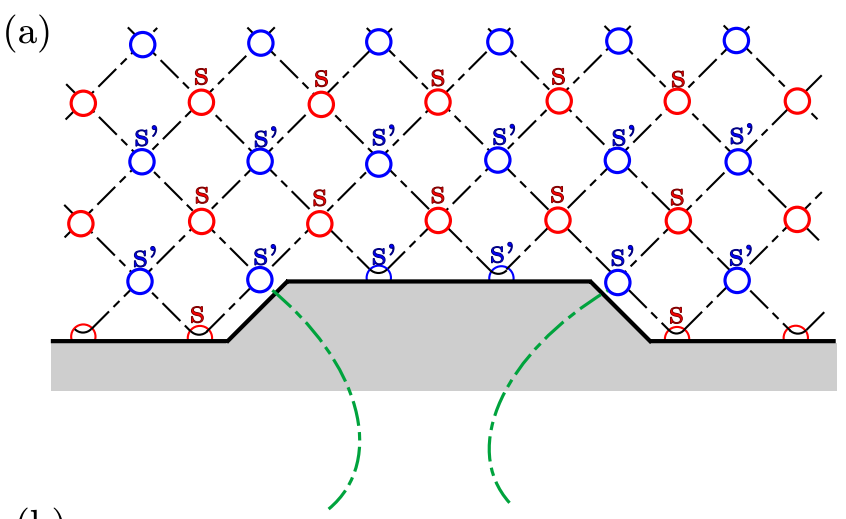

(b)

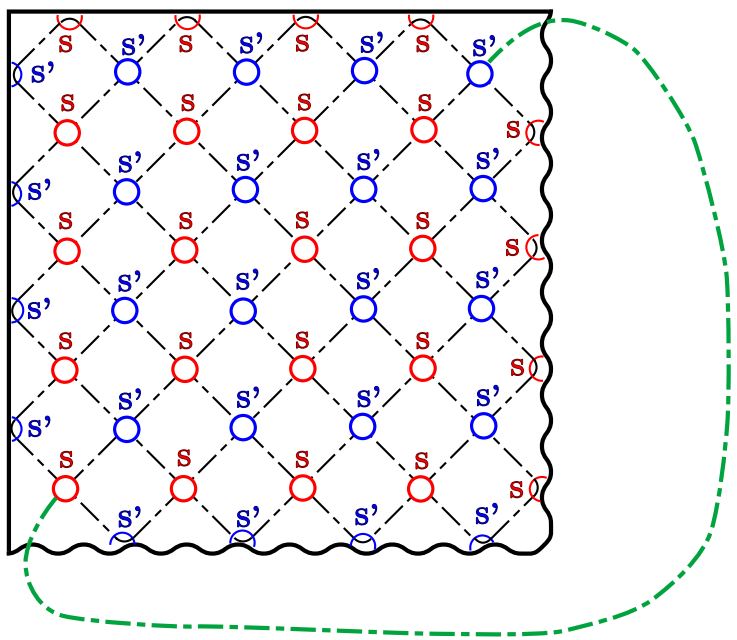

FIG. 13. (Color online) (a) A semi-infinite geometry with twopoint contacts (green dot-dashed curves) attached at the two interfaces between different types of boundaries. Each line or curve represents a Kramers doublet. (b) Closed network with mixed boundaries. The thick wavy and solid lines on the edges represent two different types of boundaries, with and without a helical edge mode at $X>X_{l}$, respectively.

to attach two-point contacts (leads) at the two interfaces between the boundary nodes of types $\mathbf{S}$ and $\mathbf{S}^{\prime}$ in order to maintain TRS. This setup is shown in Fig. 13(a). The scattering matrix that relates incoming to outgoing waves from the leads is then a $2 \times 2$ matrix. The constraint that it belongs to the symplectic symmetry class forces this matrix to be proportional to the unit $2 \times 2$ matrix up to an overall (random) phase. Hence, the two-point conductance is always unity no matter how far apart the two-point contacts are. An incoming Kramers doublet is transmitted with a probability of 1 through the disordered region in Fig. 13(b). This result may be related to the presence of a perfect transmission channel in the geometry of Fig. 3(c) by thinking of a conformal mapping that transforms a half plane with mixed boundaries to an infinite strip with mixed boundaries.

Before proceeding with the case at hand, we recall that it is expected on general grounds that the moments of the twopoint conductance in a network model at criticality, when the point contacts are far apart, decay as power laws with scaling exponents proportional to the scaling exponents $\Delta_{q}^{(\kappa)} .{ }^{35} \mathrm{Con}-$ sequently, after tuning the semi-infinite network model to criticality, the zero-dimensional boundary scaling exponents 
for the moments of $|\Psi(\boldsymbol{r})|^{2}$ with $\boldsymbol{r}$ at an interface of different types of boundaries,

$$
\Delta_{q}^{\left(0, Z_{2} \mid O\right)}=0,
$$

emerge as a signature of the nontrivial topological nature of the insulating side at the Anderson transition. We conjecture that if a description of the critical point exists in terms of a conformal field theory, exponents (6.1) might then be obtained from the correlation functions between the moments of the local operator encoding the local density of states and additional insertions of a boundary condition changing operator. ${ }^{36}$

The zero-dimensional multifractal scaling exponents [Eq. (6.1)] are different from the corner multifractal scaling exponents which characterize the scaling of the moments of wave functions at a corner with the boundary of a given type, such as the upper left corner and the lower right corner in Fig. 13(b). These corner multifractal exponents read

$$
\Delta_{q}^{(0,0)}=2 \Delta_{q}^{(1,0)}
$$

or

$$
\Delta_{q}^{\left(0, Z_{2}\right)}=2 \Delta_{q}^{\left(1, Z_{2}\right)},
$$

depending on the type of the boundary and the critical point to which the network model has been tuned. Here, Eqs. (6.2) and (6.3) follow from a general relation based on twodimensional conformal mappings that relates the scaling exponents (5.2) with $\boldsymbol{r}$ at the corner between two straight boundaries meeting to the angle $\pi / 2$ and the scaling exponents (5.2) with $\boldsymbol{r}$ along a straight boundary. ${ }^{3}$

\section{CONCLUSIONS}

We have shown that the boundary multifractal spectra for the critical normalized wave functions are sensitive to the choice of boundary conditions at the Anderson transition in the two-dimensional symplectic class. This is the first example where boundary multifractal exponents are calculated under different boundary conditions in the problem of Anderson localization-delocalization transition. It would be interesting to look for other examples of disorder-induced continuous phase transitions at which boundary critical properties depend on boundary conditions but bulk critical properties do not.

We conjecture that the two-dimensional conformal theory describing the Anderson transition in the two-dimensional symplectic universality class, if it exists, should be compatible with two distinct conformally invariant boundary conditions on the boundary of a half plane, thereby yielding two distinct sets of boundary multifractal exponents $\Delta_{q}^{(1,0)}$ and $\Delta_{q}^{\left(1, Z_{2}\right)}$. The recent observation of the QSH effect in $\mathrm{HgTe} /$ $(\mathrm{Hg}, \mathrm{Cd}) \mathrm{Te}$ quantum wells ${ }^{8}$ suggests that it might be possible to probe experimentally the Kramers degenerate edge states at criticality and the corresponding boundary multifractal spectra.

\section{ACKNOWLEDGMENTS}

This work was supported by the Next Generation Super Computing Project, Nanoscience Program, MEXT, Japan and by the National Science Foundation under Grant No. PHY05-51164. Numerical calculations were mainly performed on the RIKEN Super Combined Cluster System.
* Present address: Department of Physics, Kyoto University, Kyoto 606-8502, Japan.

${ }^{1}$ S. Hikami, A. I. Larkin, and Y. Nagaoka, Prog. Theor. Phys. 63, 707 (1980).

${ }^{2}$ Y. Asada, K. Slevin, and T. Ohtsuki, Phys. Rev. Lett. 89, 256601 (2002); Phys. Rev. B 70, 035115 (2004).

${ }^{3}$ H. Obuse, A. R. Subramaniam, A. Furusaki, I. A. Gruzberg, and A. W. W. Ludwig, Phys. Rev. Lett. 98, 156802 (2007).

${ }^{4}$ A. Mildenberger and F. Evers, Phys. Rev. B 75, 041303(R) (2007).

${ }^{5}$ C. L. Kane and E. J. Mele, Phys. Rev. Lett. 95, 146802 (2005); 95, 226801 (2005).

${ }^{6}$ B. A. Bernevig and S.-C. Zhang, Phys. Rev. Lett. 96, 106802 (2006).

${ }^{7}$ B. A. Bernevig, T. L. Hughes, and S.-C. Zhang, Science 314, 1757 (2006).

${ }^{8}$ M. König, S. Wiedmann, C. Brüne, A. Roth, H. Buhmann, L. W. Molenkamp, X.-L. Qi, and S.-C. Zhang, Science 318, 766 (2007).

${ }^{9}$ C. Wu, B. A. Bernevig, and S.-C. Zhang, Phys. Rev. Lett. 96, 106401 (2006).

${ }^{10}$ C. Xu and J. E. Moore, Phys. Rev. B 73, 045322 (2006).

${ }^{11}$ M. Onoda, Y. Avishai, and N. Nagaosa, Phys. Rev. Lett. 98, 076802 (2007).
${ }^{12}$ H. Obuse, A. Furusaki, S. Ryu, and C. Mudry, Phys. Rev. B 76, 075301 (2007).

${ }^{13}$ A. R. Subramaniam, I. A. Gruzberg, A. W. W. Ludwig, F. Evers, A. Mildenberger, and A. D. Mirlin, Phys. Rev. Lett. 96, 126802 (2006).

${ }^{14}$ A similar analysis can be done in the opposite limit of $X \rightarrow 0$, for which the topological nature of the insulating state can be deduced by considering the limit of small $\theta(>0)$. This effectively amounts to the interchange of the nodes and $\mathbf{S}$ and $\mathbf{S}^{\prime}$. (Taking the small- $\theta$ limit is justified by the phase diagram in Fig. 11 of Ref. 12.) By doing so, the conclusion as to whether a helical edge state is present on upper/lower boundaries is reversed compared to the conclusion for the $X \rightarrow \infty$ limit.

${ }^{15}$ J. T. Chalker and P. D. Coddington, J. Phys. C 21, 2665 (1988).

${ }^{16}$ Lyapunov exponents are twofold degenerate due to the Kramers degeneracy in the geometries of Figs. 3(a) and 3(b), for which the transfer matrices are $4 M \times 4 M$ matrices.

${ }^{17}$ A. MacKinnon and B. Kramer, Z. Phys. B: Condens. Matter 53, 1 (1983).

${ }^{18}$ M. R. Zirnbauer, Phys. Rev. Lett. 69, 1584 (1992).

${ }^{19}$ Y. Takane, J. Phys. Soc. Jpn. 73, 9 (2004); 73, 1430 (2004); 73, 2366 (2004).

${ }^{20}$ J. L. Cardy, J. Phys. A 17, L385 (1984).

${ }^{21}$ K. Slevin and T. Ohtsuki, Phys. Rev. Lett. 82, 382 (1999). 
${ }^{22}$ R. Klesse and M. Metzler, Europhys. Lett. 32, 229 (1995).

${ }^{23}$ M. Janssen, Int. J. Mod. Phys. B 8, 943 (1994); Phys. Rep. 295, 1 (1998).

${ }^{24}$ A. Mildenberger, A. R. Subramaniam, R. Narayanan, F. Evers, I. A. Gruzberg, and A. D. Mirlin, Phys. Rev. B 75, 094204 (2007).

${ }^{25}$ F. Evers, A. Mildenberger, and A. D. Mirlin, arXiv:0804.2334, Phys. Rev. Lett. (to be published).

${ }^{26}$ H. Obuse, A. R. Subramaniam, A. Furusaki, I. A. Gruzberg, and A. W. W. Ludwig, arXiv:0804.2409, Phys. Rev. Lett. (to be published).

${ }^{27}$ K. J. Falconer, Fractal Geometry: Mathematical Foundations and Applications (Wiley, Chichester, 1990).

${ }^{28}$ C. Mudry, C. Chamon, and X.-G. Wen, Nucl. Phys. B 466, 383 (1996).
${ }^{29}$ C. C. Chamon, C. Mudry, and X.-G. Wen, Phys. Rev. Lett. 77, 4194 (1996).

${ }^{30}$ C. Mudry, S. Ryu, and A. Furusaki, Phys. Rev. B 67, 064202 (2003).

${ }^{31}$ A. D. Mirlin and F. Evers, Phys. Rev. B 62, 7920 (2000).

${ }^{32}$ A. D. Mirlin, Y. V. Fyodorov, A. Mildenberger, and F. Evers, Phys. Rev. Lett. 97, 046803 (2006).

${ }^{33}$ B. Duplantier and A. W. W. Ludwig, Phys. Rev. Lett. 66, 247 (1991).

${ }^{34}$ T. Ando and H. Suzuura, J. Phys. Soc. Jpn. 71, 2753 (2002).

${ }^{35}$ R. Klesse and M. R. Zirnbauer, Phys. Rev. Lett. 86, 2094 (2001).

${ }^{36}$ J. L. Cardy, Nucl. Phys. B 324, 581 (1989). 